\title{
Quantitative description of hysteresis loops induced by rf radiation in long Josephson junctions
}

\author{
Olsen, Ole H.; Samuelsen, Mogens Rugholm
}

Published in:

Physical Review B

Link to article, DOI:

10.1103/PhysRevB.43.10273

Publication date:

1991

Document Version

Publisher's PDF, also known as Version of record

Link back to DTU Orbit

Citation (APA):

Olsen, O. H., \& Samuelsen, M. R. (1991). Quantitative description of hysteresis loops induced by rf radiation in long Josephson junctions. Physical Review B, 43(13), 10273-10278.

https://doi.org/10.1103/PhysRevB.43.10273

\section{General rights}

Copyright and moral rights for the publications made accessible in the public portal are retained by the authors and/or other copyright owners and it is a condition of accessing publications that users recognise and abide by the legal requirements associated with these rights.

- Users may download and print one copy of any publication from the public portal for the purpose of private study or research.

- You may not further distribute the material or use it for any profit-making activity or commercial gain

- You may freely distribute the URL identifying the publication in the public portal

If you believe that this document breaches copyright please contact us providing details, and we will remove access to the work immediately and investigate your claim. 


\title{
Quantitative description of hysteresis loops induced by rf radiation in long Josephson junctions
}

\author{
O. H. Olsen \\ NOVO-Nordisk Research Institute, NOVO allé 1, DK-2880 Bagsvard, Denmark \\ M. R. Samuelsen \\ Physics Laboratory I, Technical University of Denmark, DK-2800 Lyngby, Denmark
}

(Received 29 November 1990)

\begin{abstract}
The effect of an applied rf signal on the radiation emitted from a long Josephson junction is examined by means of a model based on the sine-Gordon equation. This system exhibits a variety of interesting phenomena, e.g., chaos and hysteresis. The hysteresis loop is examined in detail. These simple analyses show that for rf frequencies larger than a certain threshold value no hysteresis is expected. This is verified in numerical simulations where the frequency and length of the junction have been varied.
\end{abstract}

\section{INTRODUCTION}

The study of the dynamical behavior of long Josephson junctions is of fundamental as well as practical interest. From a theoretical point of view the system shows a rich variation of nonlinear properties which are suitable for detailed investigations of, e.g., nonlinear wave dynamics $^{1,2}$ and chaotic states. ${ }^{3-9}$ Further, the system is suitable for testing perturbation approaches. From an applications point of view there have been suggestions for the use of Josephson junctions in such diverse fields as microwave-oscillator amplifiers and data-processing systems. ${ }^{10,11}$

Previously, ${ }^{12-14}$ we examined the influence of an applied rf signal on the radiation emitted from a long Josephson junction by means of a model based on the sine-Gordon equation. The microwave pump signal was applied to one end of the junction. Thus the system was modeled by a perturbed one-dimensional sine-Gordon equation with appropriate boundary conditions describing the influence of the rf signal. The rms value of the voltage of the emitted signal was calculated and used to evaluate the response of the junction. Various interesting phenomena were found depending on the amplitude of the rf signal: period-doubling sequences or bifurcation trees (devil's staircase), chaos, quasiperiodicity, and hysteresis. However, only a single frequency of the applied signal and one length of the junction were considered. This system has also been considered in Refs. 8 and 9 where perturbation techniques were applied to describe chaotic dynamics of a nonequilibrium kink.

In the present paper the hysteresis loop is investigated in detail. Thus the influence of variations in frequency of the applied signal and length of the junction on the emitted radiation is examined analytically and numerically. Further, the influence of variations in the loss parameter is considered.

The hysteresis loop consists of an upper and a lower branch, respectively. The upper branch can be ascribed to a breather-mode frequency locked to the driver while the lower branch can be ascribed to a solution obtained from the linearized system. ${ }^{14}$ However, expressions can be developed describing the limits for the existence of the branches as a function of the applied frequency and damping parameter. Combination of these expressions gives a threshold value for the frequency, i.e., for larger frequencies the hysteresis loop disappears. The threshold value compares well with numerical experiments. Further, the upper branch disappears for lower values of the frequency.

The paper is outlined as follows: Section II contains a description of the model and the analytical results which can be obtained from simple energy considerations and from the knowledge about the breather solution. In Sec. III the numerical experiments and the comparisons with the analytical results are presented. Finally, Sec. IV contains the conclusion.

\section{MODEL AND ANALYTICAL RESULTS}

In this section the model used to describe the dynamical behavior of long Josephson junctions is outlined and results from simple analysis presented. The mathematical model used to describe the dynamics of a long Josephson junction is a perturbed sine-Gordon equation. The perturbation includes a dissipation term. The equation is, in normalized form,

$$
\phi_{x x}-\phi_{t t}=\sin \phi+\alpha \phi_{t},
$$

where $\phi$ is the phase difference between the two superconducting films. The spatial variable is measured in units of the Josephson penetration depth $\lambda_{J}=\left(h / 2 e d \mu_{0} J\right)^{1 / 2}$ and the time in units of the reciprocal plasma frequency $\omega_{0}^{-1}$ where $\omega_{0}=(2 e J / h C)^{1 / 2}$. Here $J$ is the Josephson current density, $d$ is the magnetic thickness of the barrier, and $C$ is the capacitance per unit area. The loss parameter $\alpha$ is defined through the relation $\alpha=G(h / 2 e J C)^{1 / 2}, G$ being the shunt conductance per unit area. The loss parameter models dissipative currents (quasiparticle currents). 
When an oscillating magnetic field $H_{e} \sin \omega t$ is applied to one end (in this case the right end) of the junction, perpendicular to the length of the junction and parallel to the plane of the barrier, the boundary conditions for the phase difference $\phi$ at the ends are

$$
\phi_{x}(0, t)=0
$$

and

$$
\phi_{x}(l, t)=a \sin \omega t,
$$

where $a=H_{e} / J \lambda_{J}$ and $l$ are the normalized magnetic field strength and junction length, respectively. Here we assume that the influence of an external magnetic field is felt only through the boundary conditions (1b), and not through Eq. (1) which describes the dynamics of the interior of the junction.

Equations (1) have been solved numerically. The numerical solution is based on a stabilized hopping scheme. ${ }^{15}$ In Fig. 1 we show the results from such a solution in terms of the rms value of $\phi_{t}$ (at $x=l$ ) as a function of the applied amplitude $a$. The specific parameter values in Fig. 1 are as follows: frequency $\omega=0.7$ and length $l=5$. The loss parameter $(\alpha=0.2)$ is held fixed. In order to simulate an experimental situation we follow the procedure from Ref. 14. Thus for $a=0$ we use flat initial conditions $\left[\phi(x, 0)=0\right.$ and $\left.\phi_{t}(x, 0)=0\right] ; a$ is then increased gradually by an amount $\Delta a$ [using a ramp function $a_{\text {new }}=a_{\text {old }}+(\Delta a / 50) t$ for $\left.t<50\right]$. The steady state is typically obtained after $t=500$; each run is continued to $t=1500$. After calculation of the last eight $\mathrm{rms}$ values of $\phi_{t}$ the computations are stopped, restarted (the initial conditions now being the previous solution), and $a$ is gradually increased. This procedure is continued for $a<2.5$ with $\Delta a=0.005$. When $a=2.5$ is reached the sign of $\Delta a$ is changed and the procedure repeated until $a=0$ is obtained. Note that the rms value of $\phi_{t}$ is defined as the square root of the following quantity:

$$
\left\langle\phi_{t}^{2}\right\rangle=\omega / 2 \pi \int_{0}^{2 \pi / \omega} \phi_{t}^{2} d t .
$$

In Fig. 1(a) the resulting curve for $\Delta a>0$ is shown, while Fig. 1(b) shows the corresponding curve for $\Delta a<0$. For each value of $a$, eight consecutive values of the rms value of $\phi_{t}$ have been plotted. This allows us to identify periodic solutions as well as solutions up to eight periods. In Fig. 1(a) it is seen that the rms value changes rapidly (from 1.14 to 1.61 ) for $a_{u, \text { th }}=1.160$ and enters another branch (referred to as the upper branch). This change corresponds to a change in the wave dynamics. For $a>1.455$ the resulting dynamics give a scattered set of dots corresponding to a chaotic time response. The response is again periodic for $1.930<a<2.160$ but enters a chaotic regime for $a>2.16$. The dependence of the dynamics on the amplitude of the applied rf signal is perhaps seen more clearly in Fig. 1(c) where we have shown the differences between the values of $\left(\left\langle\phi_{t}^{2}\right\rangle\right)^{1 / 2}$ displayed in Figs. 1(a) and 1(b). Here the periodic responses are represented as lines. Thus the regions with no hysteresis are represented by the zero line, the hysteresis loop by a line different from zero, and the chaotic time response by the sets of scattered points. We do not
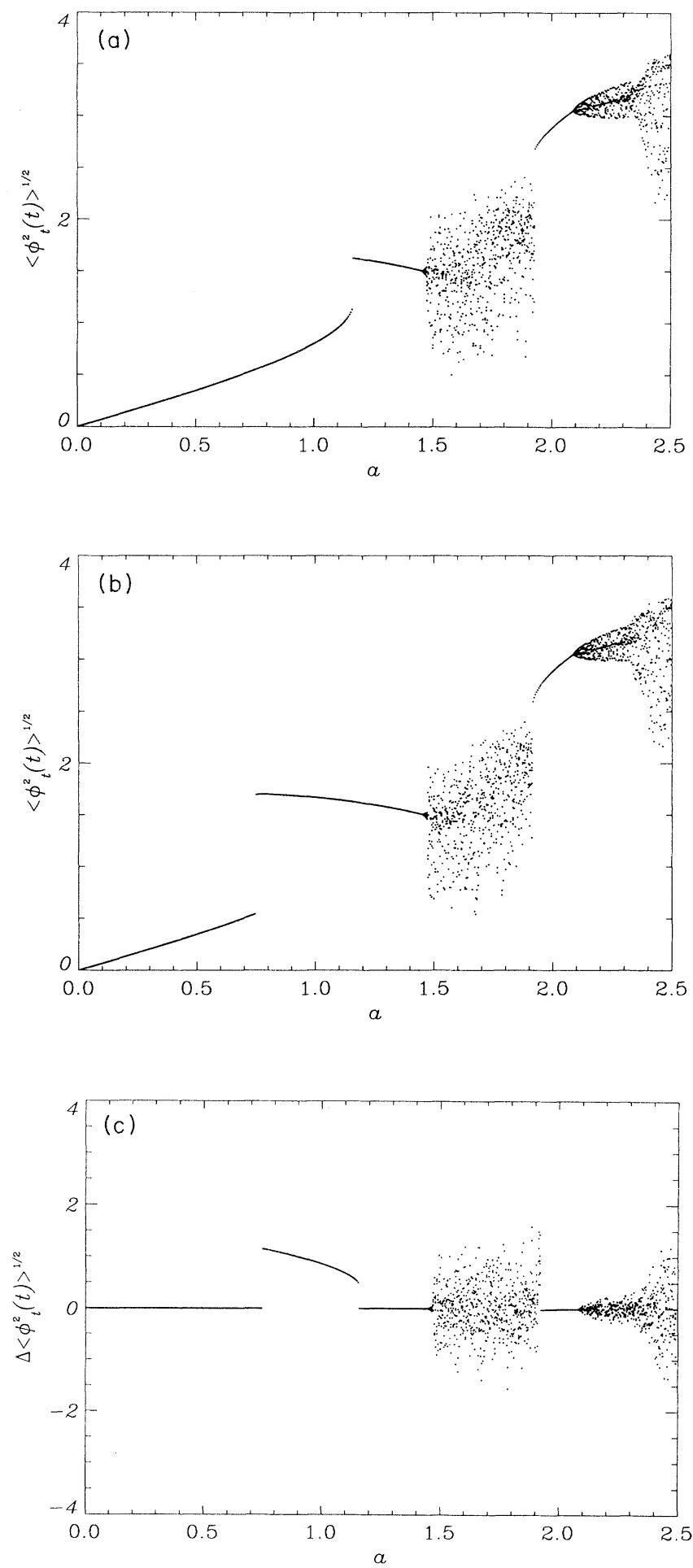

FIG. 1. The rms value $\left(\left\langle\phi_{t}^{2}\right\rangle\right)^{1 / 2}$ vs the applied amplitude $a$. Parameter values are $l=5, \alpha=0.2$, and $\omega=0.7$. In (a) the amplitude has been increased from 0 to 2.5 in steps of $\Delta a=0.005$ while (b) shows the corresponding curve for $a$ decreasing from 2.5 to 0 in steps $\Delta a=-0.005$. (c) shows the difference between the values of $\left(\left\langle\phi_{t}^{2}\right\rangle\right)^{1 / 2}$ shown in (a) and (b). The hysteresis loop is seen between $a=0.75$ and $a=1.16$, corresponding to $a_{l, \text { th }}$ and $a_{u, \mathrm{th}}$, respectively. 
intend to characterize the chaotic time response in detail in the present investigation. However, a priori we expect that low-dimensional chaos as well as turbulent behavior ${ }^{6}$ will characterize the dynamics in the scattered regions. From Fig. 1(b) it is seen that this branch can be extended to lower values of $a$. The upper branch exists down to $a_{l, \mathrm{th}}=0.750$ where the rms value decreases rapidly (from 1.70 to 0.54 ) and returns to the lower branch. In Ref. 14 the existence of the hysteresis loop was explained by identifying the modes sustaining the different branches. Thus the lower branch is sustained by a solution to the linearized equations (1a) and (1b), while the upper branch is sustained by a breather solution.

The spatial solutions sustaining the upper and lower branches are shown in Figs. 2(a) and 2(b), respectively. In Fig. 2(a) the amplitude is $a=0.75$ which is close to the threshold value for the existence of the upper branch. Here the spatial mode is well described as a breather solution. In Fig. 2(b) the amplitude has been decreased to 0.74-the spatial mode corresponds to the solution of the linearized version of Eq. (1). The solutions are indeed quite different.

In the following we give expressions for various threshold values. Note that the solution to the linearized equation fits the numerical results perfectly for lower values of the applied $a$, e.g., for $a<0.6$ in Fig. $1 .^{14}$ The mode sustaining the upper branch was identified as a breather frequency locked to the driver signal and placed symmetrically with respect to the right end of the junction. Thus the mode sustaining the upper branch is given by the breather solution
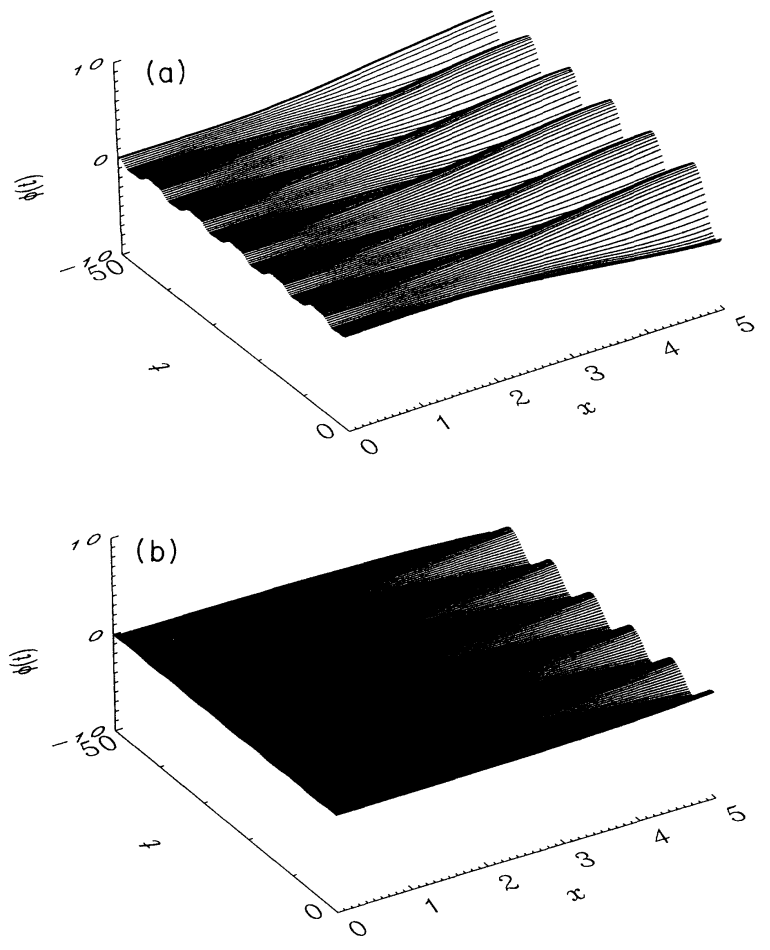

FIG. 2. The spatial solutions sustaining the upper (a) and the lower branches (b) in Fig. 1. The amplitudes of the applied rf signals are 0.75 (a) and 0.74 (b). Time evolution has been followed in 50 time units.

$$
\phi(x, t)=4 \tan ^{-1}\left\{\left[\left(1-\omega^{2}\right)^{1 / 2} / \omega\right] \sin \left(\omega t+\theta_{\mathrm{br}}\right) / \cosh \left[\left(1-\omega^{2}\right)^{1 / 2}(x-1)\right]\right\}
$$

In order to determine an expression for the lower threshold value $a_{l, \text { th }}$ for the upper branch we apply the usual perturbation approach. ${ }^{1}$ Define the energy

$$
H=\int_{0}^{1}\left[\frac{1}{2} \phi_{x}^{2}+\frac{1}{2} \phi_{t}^{2}+(1-\cos \phi)\right] d x .
$$

Time differentiation of $H$ and use of Eq. (1) yields

$$
d H / d t=-\alpha \int_{0}^{l} \phi_{t}^{2} d x+\left.\phi_{x} \phi_{t}\right|_{0} ^{l}
$$

Here the first term represents energy dissipation while the latter term represents energy input. In one period of oscillation the energy change is

$$
\Delta H=-\alpha \int_{0}^{2 \pi / \omega} \int_{0}^{l} \phi_{t}^{2} d x d t+\left.\int_{0}^{2 \pi / \omega} \phi_{x} \phi_{t}\right|_{x=l} d t .
$$

In a stationary situation the energy change in one period of oscillation is zero. Close to the threshold value the energy input is at a maximum, forcing the phase angle $\theta=0$ in Eq. (2). Insertion of the breather solution in Eq. (5) yields ${ }^{11}$

$$
\Delta H=-16 \pi a \sin ^{-1}\left(1-\omega^{2}\right)^{1 / 2}+8 \pi[(1-\omega) /(1+\omega)]^{1 / 2} .
$$

Thus for $\Delta H=0$ we get an expression for the lower threshold value

$$
a_{l, \mathrm{th}}=2 \alpha[(1+\omega) /(1-\omega)]^{1 / 2} \sin ^{-1}\left(1-\omega^{2}\right)^{1 / 2} .
$$

Now, an expression for the upper threshold, $a_{u \text {, th }}$, can be given applying the following argument. The solution sustaining the lower branch can be found linearizing Eq. (1). When the amplitude $a$ exceeds the maximum value of the spatial derivative of the breather solution corresponding to the applied frequency a switch from the lower branch to upper branch occurs. The maximum spatial derivative for a breather of frequency $\omega$ is

$$
a_{u, \mathrm{th}}=2\left(1-\omega^{2}\right)
$$

From Eqs. (7) and (8) it is seen that the hysteresis phenomenon will disappear for large values of the fre- 
quency, i.e., for values of $\omega$ where $a_{u, \mathrm{th}}<a_{l, \mathrm{th}}$. Thus the following relation between the loss parameter and the frequency gives the upper value of $\omega$ for the existence of the hysteresis loop:

$$
\alpha \sin ^{-1}\left(1-\omega^{2}\right)^{1 / 2}=\left[\frac{(1-\omega)^{3}}{1+\omega}\right]^{1 / 2}
$$

In Fig. 3 the relations for $a_{l, \text { th }}$ and $a_{u, \text { th }}$, Eqs. (7) and (8), are shown as a function of the frequency $\omega$. The points are obtained from a full simulation of Eq. (1). In the next section the numerical results will be discussed.
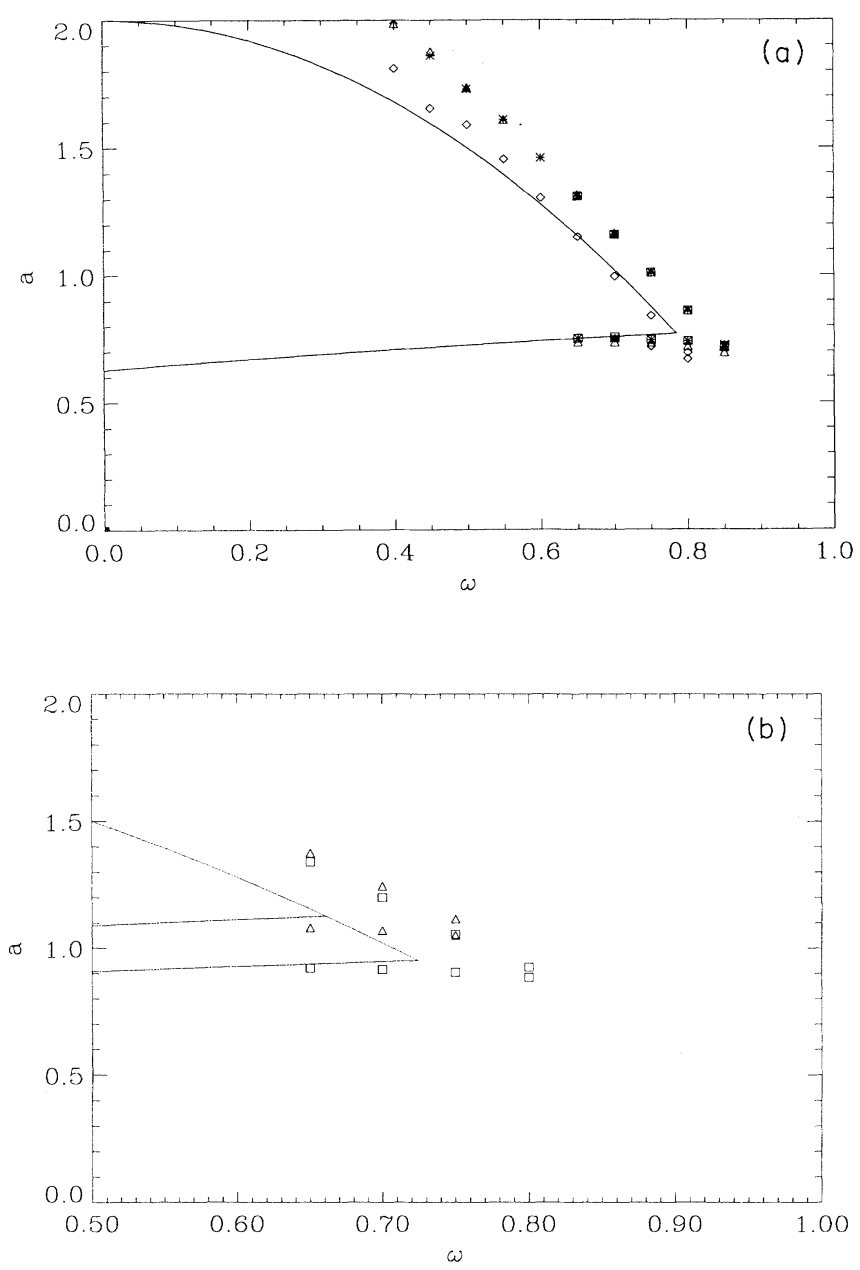

FIG. 3. Comparison between the analytical expressions for the threshold values and numerical results for $\alpha=0.2$ (a). The solid curves are Eq. (7) (lower) and (8) (upper), respectively. The points are obtained numerically and various lengths have been considered: $l=2.5$ (diamonds), $l=5$ (squares), $l=7.5$ (asterisks), and $l=10$ (triangles). In (b) a comparison is shown between the analytical expressions for the threshold values and numerical results for $\alpha=0.25$ (squares) and $\alpha=0.3$ (triangles). The solid curves are obtained from the analysis.

\section{NUMERICAL RESULTS}

In this section we compare the analytical expressions for the various threshold values obtained in the preceding section. Thus we have examined the influence of various parameters (frequency, length of junction, and loss parameter) on the width of the hysteresis loop. Further, we show some examples of the dependence of $\left(\left\langle\phi_{t}^{2}\right\rangle\right)^{1 / 2}$ on the applied amplitude $a$.

\section{A. Analytical versus numerical results}

In order to compare the analytical results and the numerical simulations we have performed a set of numerical experiments like the one resulting in Fig. 1. The solid curves in Fig. 3(a) are obtained from Eq. (7) (lower) and Eq. (8) (upper). For a certain value of $\omega$ the distance between the curves gives the width of the hysteresis loop, i.e., $a_{l, \mathrm{th}}-a_{u \text {, th }}$. For values of $\omega$ larger than the value corresponding to the intersection of the curves [corresponding to Eq. (9)] no hysteresis is expected. The loss parameter in Fig. 3(a) is $\alpha=0.2$. For this value of the parameter we have determined $a_{l, \text { th }}$ and $a_{u \text {, th }}$ for $l=2.5,5.0,7.5$ and 10.0; see Fig. 3(a). In accordance with the analysis in the preceding section we see that the hysteresis loop disappears for $\omega$ larger than a certain value. However, for values of $\omega<0.55$ the hysteresis loop disappears as well. This is surprising and seems independent of the length of the junction and the value of the loss parameter. Further, the threshold values determined numerically are independent of the length of the junction for $l>5$.

From Eq. (7) it is seen that $a_{l, \text { th }}$ scales with the loss parameter. In Fig. 3(b) we have shown the threshold values obtained analytically and numerically for $\alpha=0.25$ and 0.3 . Again we see that the hysteresis loop disappears for large values of $\omega$, but also for smaller values of $\omega$. In general a good agreement between the threshold value [Eq. (7)] and the numerical results is observed.

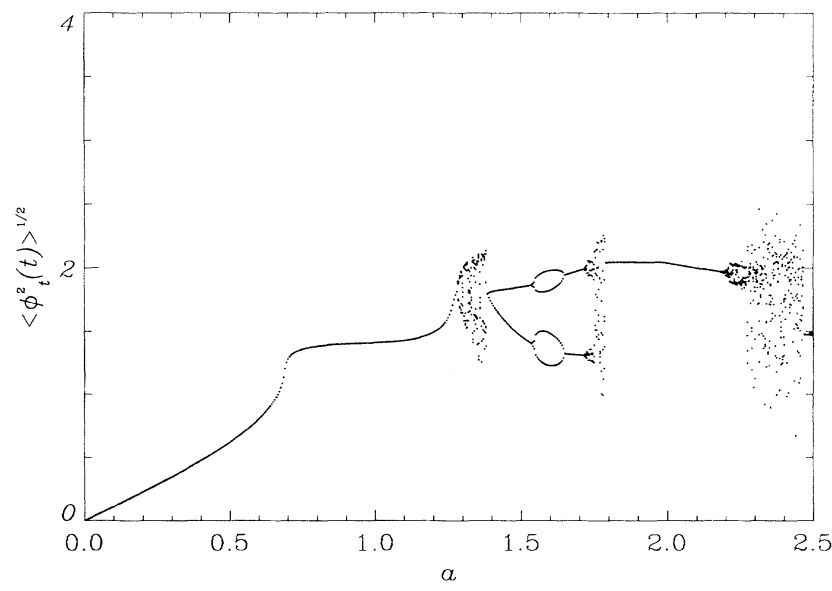

FIG. 4. The rms value $\left(\left\langle\phi_{t}^{2}\right\rangle\right)^{1 / 2}$ vs the applied amplitude $a$ for $l=5, \omega=0.87, \alpha=0.2$, and $a$ has been increased in steps of 0.005 . Note that this value of $\omega$ is right above the threshold value for the existence of the hysteresis loop. 


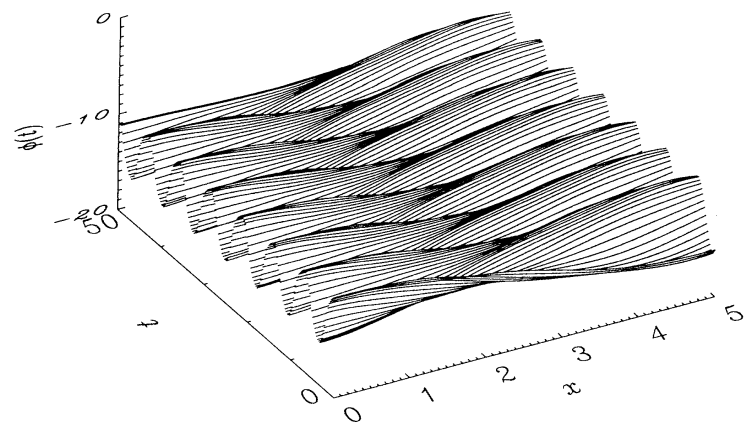

FIG. 5. The spatial pattern representing the periodic solution for $l=5, \omega=0.87$, and $\alpha=0.2$ (see Fig. 4).

\section{B. Examples of interplay between frequency and amplitude of $\mathrm{rf}$ signal}

Apart from the hysteresis loops discussed above, which was ascribed to the existence of two different spatial patterns, we also find other interesting responses. However, these are difficult to explain in detail and the results will therefore just be shown and supplied with some comments.

In Fig. 4 the rms value $\left(\left\langle\phi_{t}^{2}\right\rangle\right)^{1 / 2}$ is shown as a function of the applied amplitude $a$ for $\omega=0.87$. For this value of $\omega$ we are just above the threshold for the existence of the hysteresis loop. For $a>1.280$ the response is irregular judged from the scattering of points in that region. However, the irregular response is caused by a change from a single periodic response to a doubleperiodic solution which in turn is bifurcated into a fourperiod solution. This solution is converted back to a single periodic solution through the reverse sequence. Again for $a>1.715$ a region of scattered points is observed, which denotes a transition to a one-period solution. For $a>2.190$ the response is again represented by a set of scattered points. The spatial pattern corresponding to the single periodic solution for $1.790<a<2.190$ is shown in Fig. 5. Compared to the patterns sustaining the hysteresis loops we find this pattern substantially different. The pattern is almost symmetrical with respect to the middle of the junction.

In Fig. 6 we show the results of a numerical experiment for $\omega=0.55$. For this value of $\omega$ the hysteresis loop does not exist. However, for $\omega=0.57$ the hysteresis loop still exists and the threshold values are in good agreement with the analytical results presented in the preceding sec-

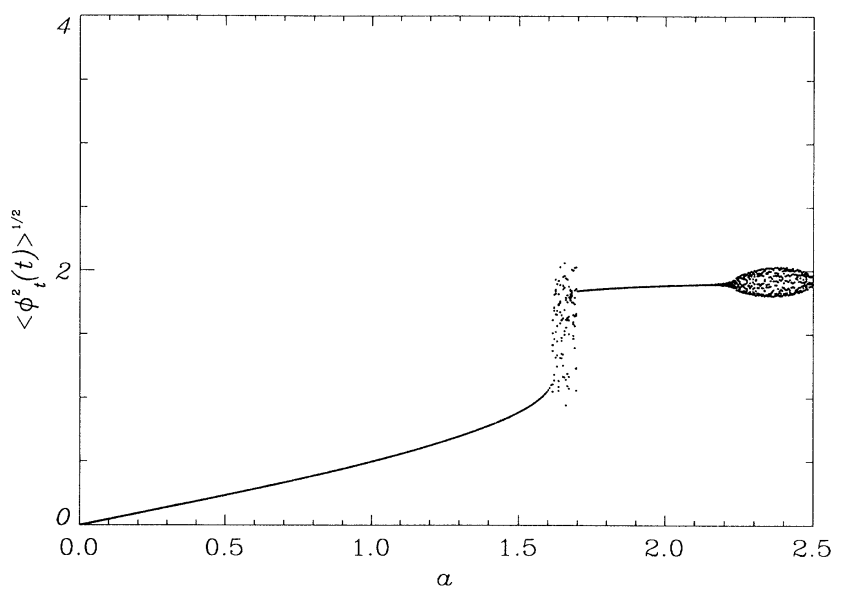

FIG. 6. The rms value $\left(\left\langle\phi_{t}^{2}\right\rangle\right)^{1 / 2}$ vs the applied amplitude $a$ for $l=5, \omega=0.55, \alpha=0.2$, and $a$ has been increased in steps of 0.005 . Note that for this value of $\omega$ (and for smaller values) the hysteresis loop disappears.

tion. So far we have no explanation for the disappearance of the hysteresis loop. This will be a subject for future investigations.

Finally in this section we note that apart from the results shown above we have observed a variety of responses to variations in frequency and amplitude of the applied rf signal. This will be discussed elsewhere.

\section{CONCLUSION}

In the present paper the effect of an applied rf signal on the radiation emitted from a long Josephson junction has been examined by means of a model based on the sineGordon equation. We have in particular focused on the existence of hysteresis loops. The hysteresis loop has been examined in detail. These simple analyses show that for $\mathrm{rf}$ frequencies larger than a certain threshold value no hysteresis is expected. This is verified in numerical simulations where the frequency and length of the junction have been varied. Further, the upper threshold value for the existence of the loop is well reproduced in the numerical experiments. However, the hysteresis loop disappears for $\omega<0.56$. This will be a target for further investigations.

\section{ACKNOWLEDGMENTS}

L. Guerrero is thanked for helpful discussions.
${ }^{1}$ D. W. McLaughlin and A. C. Scott, Phys. Rev. A 18, 1652 (1978).

${ }^{2}$ P. S. Lomdahl, O. H. Sørensen, and P. L. Christiansen, Phys. Rev. B 25, 5737 (1982).

${ }^{3}$ A. R. Bishop, K. Fesser, P. S. Lomdahl, and S. E. Trullinger, Physica D 7, 259 (1983).
${ }^{4}$ A. R. Bishop, K. Fesser, P. S. Lomdahl, W. C. Kerr, M. B. Williams, and S. E. Trullinger, Phys. Rev. Lett. 50, 1095 (1983).

${ }^{5}$ P. S. Lomdahl and M. R. Samuelsen, Phys. Rev. B 34, 664 (1986).

${ }^{6}$ M. Octavio and L. E. Guerrero, Phys. Rev. A 42, 4630 (1990). 
${ }^{7}$ L. E. Guerrero and M. Octavio, Phys. Rev. A 37, 3641 (1988).

${ }^{8}$ Y. S. Kivshar and B. A. Malomed, Rev. Mod. Phys. 61, 762 (1989).

${ }^{9}$ B. A. Malomed, Phys. Lett. 123A, 494 (1987).

${ }^{10}$ R. Monaco, S. Pagano, and G. Costabile, Phys. Lett. 131A, 122 (1988).

${ }^{11}$ A. Barone and G. Paterno, Physics and Applications of the Josephson Effect (Wiley, New York, 1982).
${ }^{12}$ O. H. Olsen and M. R. Samuelsen, Appl. Phys. Lett. 47, 1007 (1985).

${ }^{13}$ O. H. Olsen and M. R. Samuelsen, Phys. Lett. 119, 391 (1987).

${ }^{14}$ O. H. Olsen and M. R. Samuelsen, Phys. Rev. B 34, 3510 (1986).

${ }^{15}$ R. K. Dodd, J. C. Eilbeck, J. D. Gibbon, and H. C. Morris, Solitons and Nonlinear Wave Equations (Academic, London, 1982), p. 581. 